\title{
Making simple changes to the way a starchy carbohydrate meal is prepared can significantly reduce the postprandial glucose response
}

\author{
T.M. Robertson, J.E. Brown and M.D. Robertson \\ University of Surrey, Guildford, GU2 $7 X H$
}

Resistant starches (RS) are classed as a dietary fibre because they are not digested and absorbed in the small intestine, instead they pass to the colon where they are metabolised by colonic bacteria. Consequently, they do not contribute to the postprandial glucose response. Replacing some of the available carbohydrate $(\mathrm{CHO})$ in a meal, with $\mathrm{RS}$, has been demonstrated to reduce postprandial glycaemia $^{(1)}$. Furthermore, different domestic rice cooking methods result in differing amounts of RS formation, in vitro ${ }^{(2)}$. It is not known whether this translates into an effect on postprandial glucose metabolism, in vivo.

This study investigated whether changing the way a starchy carbohydrate meal was prepared would affect the postprandial glucose response. Ten healthy participants were recruited to this randomised crossover study. At each study visit they consumed an identical pasta meal (100 g pasta, $75 \mathrm{~g}$ tomato sauce and $10 \mathrm{~g}$ olive oil, providing $486 \mathrm{kcal}$ energy, $77 \mathrm{~g}$ CHO, $13 \mathrm{~g}$ fat and $14 \mathrm{~g}$ protein) prepared in one of three ways: freshly cooked, chilled and consumed cold or chilled and reheated (microwave). All meals were consumed by all participants over the course of their three visits. Capillary blood samples were taken for $2 \mathrm{hr}$ following the meal for subsequent glucose analysis.

Repeated measures ANOVA on postprandial glucose time-point data found a significant difference between meals $(\mathrm{p}<0.01$, Fig. 1), with $\sim 1 \mathrm{mmol} / 1$ reduction observed in peak glucose and $41 \%$ reduction in glucose incremental area under the curve between freshly cooked and chilled/reheated meals.

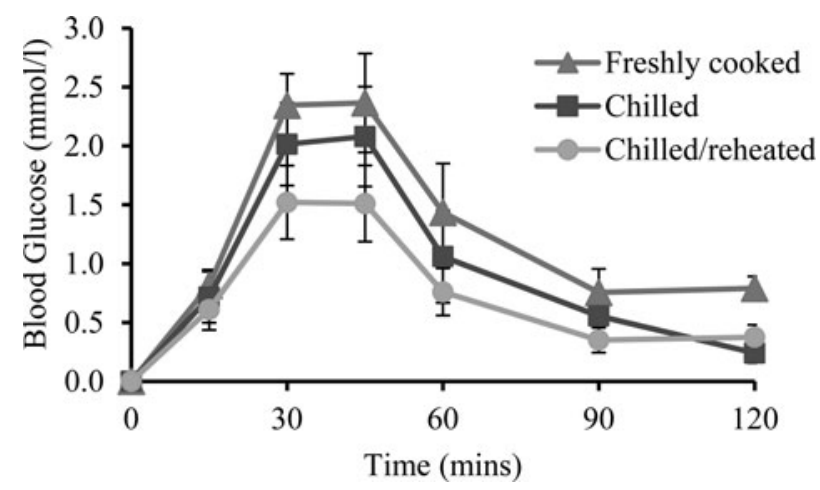

Fig. 1. Change in postprandial glucose response following consumption of the same pasta meal, prepared in three different ways, freshly cooked, chilled or chilled and reheated.

It is hypothesised that the greatest reduction, observed in the chilled/reheated meal, is a result of two types of RS formation in that meal; RS3 is formed by starch retrogradation when chilled, whereas RS5 is formed when a starchy carbohydrate is heated in the presence of lipid. However, this is still unproven as RS measurement was outside the scope of this pilot study. Nevertheless, these results demonstrate that making simple changes to food preparation methods, such as can easily be achieved in a domestic kitchen, can significantly reduce the postprandial glucose excursion. With the prevalence of diabetes increasing, the ability to change the health impact of the foods we eat, without changing the food itself, is of high importance.

1. Behall KM, Scholfield DJ, Hallfrisch JG et al. (2006) Diabetes Care 29(5), 976-81.

2. Reed MO, Ai Y, Leutcher JL et al. (2013) J Food Sci 78(7), H1076-81. 